International Journal of Pure and Applied Mathematics

Volume 85 No. 1 2013, 69-81

ISSN: 1311-8080 (printed version); ISSN: 1314-3395 (on-line version)

url: http://www.ijpam.eu

doi: http://dx.doi.org/10.12732/ijpam.v85i1.6

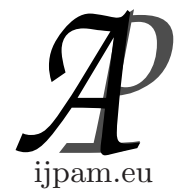

\title{
THE THREE-COLOR TRIANGLE PROBLEM
}

\author{
Yutaka Nishiyama \\ Department of Business Information \\ Faculty of Information Management \\ Osaka University of Economics \\ 2, Osumi Higashiyodogawa Osaka, 533-8533, JAPAN
}

\begin{abstract}
Place elements in the form of an inverted triangle, and color them from top to bottom by row according to some rule. When doing so, is it possible to predict the color of the element that becomes the triangle's bottom vertex? It turns out that when you begin with a triangle of $4,10,28$, c elements in its top row, knowing the colors of that row's leftmost and rightmost elements is sufficient to predict the color of that bottom vertex, and this article presents an elegant proof of this surprising result. Coloring triangles is simple even for children, but this problem provides a springboard to learning about some advanced mathematics, including Abelian groups, the superposition principle, and fractal structures.
\end{abstract}

AMS Subject Classification: 00A08, 20K01, 26B40, 28A80

Key Words: congruence, Abelian group, superposition principle, fractal

\section{Mysterious Triangles}

My British friend Steve Humble sent me a curious problem. Say that you have an inverted triangle like the one shown in Fig. 1. The top row has 10 elements, each randomly assigned one of three colors (we'll use black, gray, and white).

Received: December 21, 2012

(c) 2013 Academic Publications, Ltd. url: www.acadpubl.eu 
The second row is colored according to the following rules:

1) If the two elements above a given element are the same color, the lower element takes that color.

2) If the two elements above a given element are different colors, the lower element takes the third color.

The problem posed is this: For a triangle created by repeating these rules for each row, is it possible to predict the color of the final element, which forms the triangle's lower vertex?

As it turns out this is indeed possible, and surprisingly all you need to know is the colors of the leftmost and rightmost elements of the top row. In the example of Fig. 1, the leftmost element is white and the rightmost one is gray. Applying the two rules above to use these elements to predict the color of the final element results in gblack,h and indeed that is the case here. Since there are 10 elements in the top row there are $3^{10}=59,049$ possible color patterns, but Steve's bold claim was that the patterns the top row generates on its way down do not ultimately affect the color of the final element.

Only half believing him, I tried my hand at generating a few triangles myself. Letting the number of elements in the top row be $n$, it was clear enough that Steve's assertion held for the $n=2$ case, and some quick scribbling showed that $n=4$ works as well. One trial of the $n=10$ case had worked, but manually verifying all 59,049 patterns was out of the question. Turning to the power of computers, I wrote a simple program that attempted each possible pattern, and sure enough the rule worked for each one.

Having established that the rules could be applied to triangles where $n$ is 2,4 , and 10, I became curious as to what other numbers might work. Some modification of my program turned up $n=28$ as another solution. (If only I had realized at this point that the sequence $n=2,4,10,28, \cdots$ could also be represented as $3^{s}+1$ for $\left.s=0,1, \cdots\right)$ Another curious result was that for the other cases, where $n=3,5,6,7, \cdots$, the rule held $1 / 3$ of the time, but did not hold $2 / 3$ of the time. As to why that happens, I am still not sure.

\section{Abelian Groups}

I had used brute force to check all 59,049 cases for $n=10$, but that's not very elegant. The $n=4$ and $n=10$ cases should work by the same principle, so let's look at a more graceful approach to the $n=4$ case.

Taking our three colors as black, gray, and white, choose one to use as a base (in this discussion, we'll use white). Next we assign numbers to each 


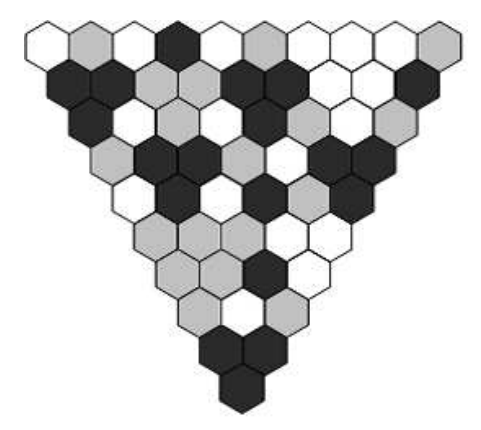

Figure 1: An inverted triangle with colored elements
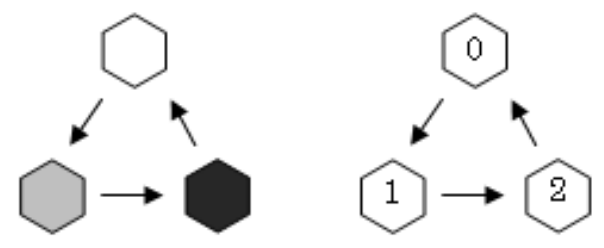

$\bmod 3$

Figure 2: Cycling through three colors

color, for example 0 for white, 1 for gray, and 2 for black. We'll say that white follows black, so cycling through the colors would be white, gray, black, white, gray, black, or numerically speaking $0,1,2,0,1,2, \cdots$, We're only using three numbers, so you can think of this as a mod 3 cycle (Fig. 2).

Now let's organize the coloring rules in a table. Our rules are that white and white give white, gray and gray give gray, black and black give black, white and gray give black, gray and black give white, and black and white give gray. Table 1 summarizes this, and if we think of combining colors as an operation, we can see that the set (white, gray, black) is closed for the operation and forms a commutative group. In other words, the coloring operation forms an example of an Abelian group.

The rows in the inverted triangle have $4,3,2$, then 1 elements, and we can denote an element as $a_{j}^{i}$, where $i$ is the row number (from the top) and $j$ is the element's position (from the left). So in the case of $n=4$, the elements in the top row are $\left(a_{1}^{1}, a_{2}^{1}, a_{3}^{1}, a_{4}^{1}\right)$, those in the second row are $\left(a_{1}^{2}, a_{2}^{2}, a_{3}^{2}\right)$, those in the third row are $\left(a_{1}^{3}, a_{2}^{3}\right)$, and the element in the bottom row is $\left(a_{1}^{4}\right)$.

Assume that all the elements in the top row are white. That would make 


\begin{tabular}{|c|c|c|c|}
\hline & W & $\mathrm{G}$ & B \\
\hline $\mathrm{W}$ & $\overline{\mathrm{W}}$ & B & $\mathrm{G}$ \\
\hline $\mathrm{G}$ & $\bar{B}$ & $\bar{G}$ & $\overline{\mathrm{W}}$ \\
\hline B & $\bar{G}$ & $\overline{\mathrm{W}}$ & $\mathrm{B}$ \\
\hline
\end{tabular}

\begin{tabular}{l|l|l|l|}
\multicolumn{1}{c}{} & \multicolumn{1}{c}{0} & 1 & \multicolumn{1}{c}{2} \\
\cline { 2 - 4 } 0 & 0 & 2 & 1 \\
\cline { 2 - 4 } 1 & 2 & 1 & 0 \\
\cline { 2 - 4 } 2 & 1 & 0 & 2 \\
\cline { 2 - 4 } & & &
\end{tabular}

Table 1: Colors as an Abelian (commutative) group
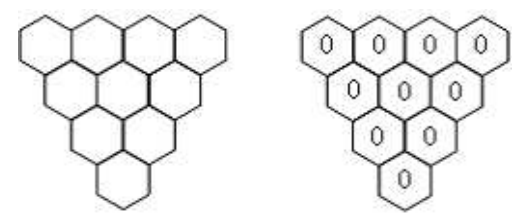

Figure 3: A triangle with all white elements in the top row

each element in the second and third row also white, and so the final element. The leftmost and rightmost elements in the top row are white, as is the bottom vertex, which follows the rules. Describing this in equations, we have

$$
\begin{aligned}
& \left(a_{1}^{1}, a_{2}^{1}, a_{3}^{1}, a_{4}^{1}\right)=(0,0,0,0) \\
& \left(a_{1}^{2}, a_{2}^{2}, a_{3}^{2}\right)=(0,0,0) \\
& \left(a_{1}^{3}, a_{2}^{3}\right)=(0,0) \\
& \left(a_{1}^{4}\right)=(0)
\end{aligned}
$$

with the final row $\left(a_{1}^{4}\right)=(0)$ predicted by the leftmost and rightmost elements $\left(a_{1}^{1}, a_{4}^{1}\right)=(0,0)$ of the topmost row (Fig. 3).

\section{Basic Patterns}

In the $n=4$ case, there are $3^{4}=81$ possible coloring patterns, but these can be broken down into the following four basic patterns. Put another way, four basic patterns can generate all possible coloring patterns. Let's see how.

Pattern 1 begins with (gray, white, white, white) as its first row, followed by (black, white, white), then (gray, white), and ending with (black).

$$
\begin{aligned}
& \left(a_{1}^{1}, a_{2}^{1}, a_{3}^{1}, a_{4}^{1}\right)=(1,0,0,0) \\
& \left(a_{1}^{2}, a_{2}^{2}, a_{3}^{2}\right)=(2,0,0) \\
& \left(a_{1}^{3}, a_{2}^{3}\right)=(1,0) \\
& \left(a_{1}^{4}\right)=(2)
\end{aligned}
$$



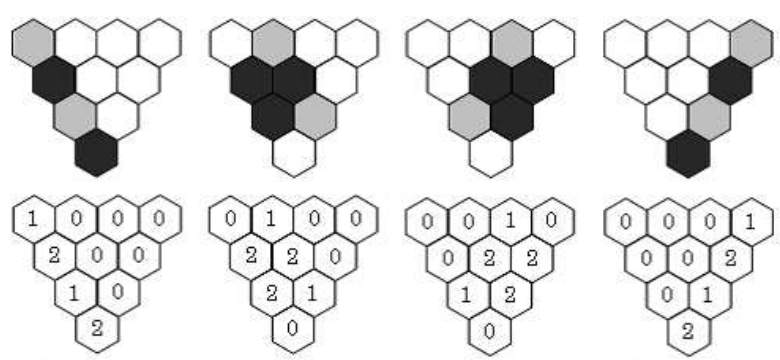

Basic pattern 1 Basic pattern 2 Basic pattern 3 Basic pattern 4

Figure 4: The four basic patterns for $n=4$.

Pattern 2 begins with (white, gray, white, white) as its first row, followed by (black, black, white), then (black, gray), and ending with (white).

$$
\begin{aligned}
& \left(a_{1}^{1}, a_{2}^{1}, a_{3}^{1}, a_{4}^{1}\right)=(0,1,0,0) \\
& \left(a_{1}^{2}, a_{2}^{2}, a_{3}^{2}\right)=(2,2,0) \\
& \left(a_{1}^{3}, a_{2}^{3}\right)=(2,1) \\
& \left(a_{1}^{4}\right)=(0)
\end{aligned}
$$

Pattern 3 begins with (white, white, gray, white) as its first row, followed by (white, black, black), then (gray, black), and ending with (white).

$$
\begin{aligned}
& \left(a_{1}^{1}, a_{2}^{1}, a_{3}^{1}, a_{4}^{1}\right)=(0,0,1,0) \\
& \left(a_{1}^{2}, a_{2}^{2}, a_{3}^{2}\right)=(0,2,2) \\
& \left(a_{1}^{3}, a_{2}^{3}\right)=(1,2) \\
& \left(a_{1}^{4}\right)=(0)
\end{aligned}
$$

Pattern 4 begins with (white, white, white, gray) as its first row, followed by (white, white, black), then (white, gray), and ending with (black).

$$
\begin{aligned}
& \left(a_{1}^{1}, a_{2}^{1}, a_{3}^{1}, a_{4}^{1}\right)=(0,0,0,1) \\
& \left(a_{1}^{2}, a_{2}^{2}, a_{3}^{2}\right)=(0,0,2) \\
& \left(a_{1}^{3}, a_{2}^{3}\right)=(0,1) \\
& \left(a_{1}^{4}\right)=(2)
\end{aligned}
$$

Each of these four basic patterns follows the rules and prediction, with the color of the bottom vertex determined by the color of the leftmost and rightmost elements in the first row (Fig. 4). 


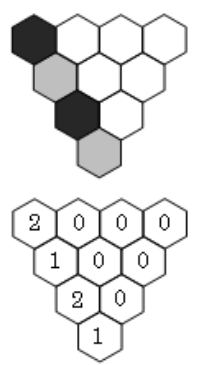

(1)

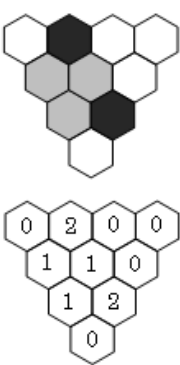

(2)
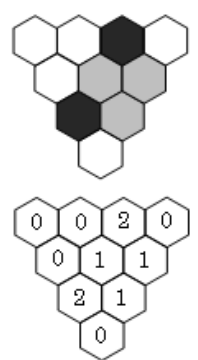

(3)
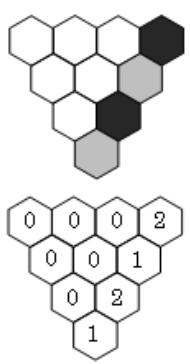

(4)

Figure 5: Modifications of the basic patterns

\section{Modifying the Four Basic Patterns}

We can modify these four basic patterns by changing the gray element in each top row to black. For example, if we make the top row (black, white, white, white), then the second row becomes (gray, white, white), the third row becomes (black, white), and the bottom vertex thus becomes (gray).

We can also do this using numerical calculation, for example by doubling each of the elements in the first pattern of Fig. 4, and assigning the remainder after division by 3 in cases where doubling gave a number larger than 2 . In other words, calculations are performed $\bmod 3$.

$$
\begin{aligned}
& \left(a_{1}^{1}, a_{2}^{1}, a_{3}^{1}, a_{4}^{1}\right)=(1,0,0,0) \quad 2=(2,0,0,0)(2,0,0,0)(\bmod 3) \\
& \left(a_{1}^{2}, a_{2}^{2}, a_{3}^{2}\right)=(2,0,0) \quad 2=(4,0,0)(1,0,0)(\bmod 3) \\
& \left(a_{1}^{3}, a_{2}^{3}\right)=(1,0) \quad 2=(2,0)(2,0)(\bmod 3) \\
& \left(a_{1}^{4}\right)=(2) \quad 2=(4)(1)(\bmod 3)
\end{aligned}
$$

In a similar way, we can create triangles that begin with (white, black, white, white), (white, white, black, white), and (white, white, white, black) (Figs. 5(1)-(4)).

\section{Superpositions}

Let's show how to generate each of the $3^{4}=81$ possible patterns for $n=4$ using the four basic patterns.

Example 1. Consider the case of white elements with some gray elements mixed in. For example, suppose that two gray elements are added in the pattern 


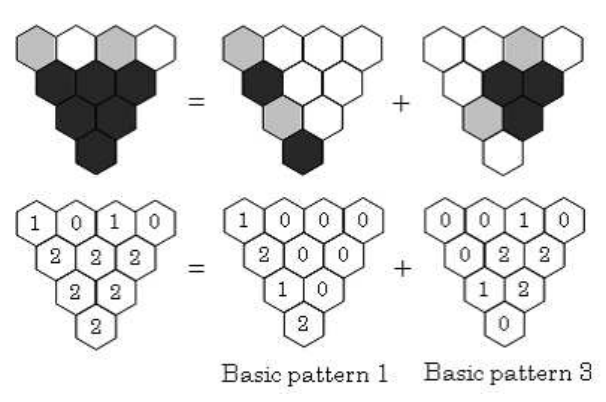

Figure 6: Superposition

(gray, white, gray, white). We can think of this as a combination of pattern 1 (gray, white, white, white) and pattern 3 (white, white, gray, white), created by adding the respective elements of each row (See Fig. 6):

$$
\begin{aligned}
& \left(a_{1}^{1}, a_{2}^{1}, a_{3}^{1}, a_{4}^{1}\right)=(1,0,0,0)+(0,0,1,0)=(1,0,1,0) \\
& \left(a_{1}^{2}, a_{2}^{2}, a_{3}^{2}\right)=(2,0,0)+(0,2,2)=(2,2,2) \\
& \left(a_{1}^{3}, a_{2}^{3}\right)=(1,0)+(1,2)=(2,2) \\
& \left(a_{1}^{4}\right)=(2)+(0)=(2)
\end{aligned}
$$

Such combinations of the basic patterns follow the superposition principle, in the same way as when combining sine waves. The basic patterns are mutually independent, and operate independently with regard to combinations. You can think of the four basic patterns as sine waves that are out of phase, or of different frequencies, and you can think of our modification as doubling each wave's amplitude. This sine-like application of the superposition principle allows simple addition of the elements.

Example 2 (All Grays). We looked at an example of superimposing two basic patterns, but now let's see how combining all four basic patterns turns everything gray (Fig. 7), something akin to simultaneously playing multiple sound frequencies to create white noise. 3)

$$
\left(a_{1}^{1}, a_{2}^{1}, a_{3}^{1}, a_{4}^{1}\right)=(1,0,0,0)+(0,1,0,0)+(0,0,1,0)+(0,0,0,1)=(1,1,1,1)(1,1,1,1)(\bmod
$$

$$
\begin{aligned}
& \left(a_{1}^{2}, a_{2}^{2}, a_{3}^{2}\right)=(2,0,0)+(2,2,0)+(0,2,2)+(0,0,2)=(4,4,4)(1,1,1)(\bmod 3) \\
& \left(a_{1}^{3}, a_{2}^{3}\right)=(1,0)+(2,1)+(1,2)+(0,1)=(4,4)(1,1)(\bmod 3) \\
& \left(a_{1}^{4}\right)=(2)+(0)+(0)+(2)=(4)(1)(\bmod 3)
\end{aligned}
$$




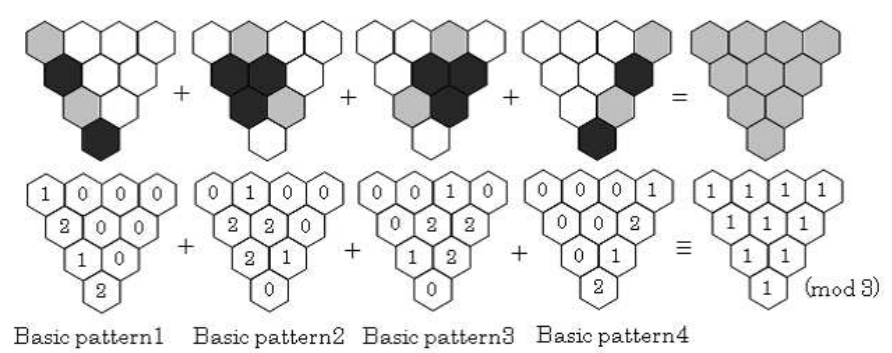

Figure 7: All grays

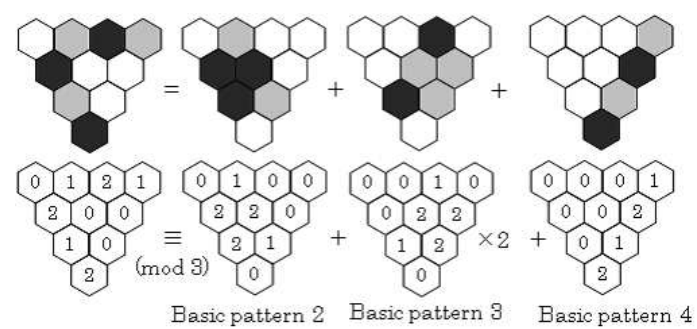

Figure 8: Adding gray and black to white

Doubling each element results in an all-black pattern, something I urge readers to try for themselves.

Example 3 (Adding Gray and Black to White). Let's consider the case of adding gray and black to white, in other words, a triangle that uses all the colors. A top row of (white, gray, black, gray) can be created by superimposing basic pattern 2 (white, gray, white, white), a doubled basic pattern 3 (white, white, gray, white), and basic pattern 4 (white, white, white, gray). Writing out equations, we get the following:

$$
\begin{aligned}
& \left(a_{1}^{1}, a_{2}^{1}, a_{3}^{1}, a_{4}^{1}\right)=(0,1,0,0)+(0,0,1,0) 2+(0,0,0,1)=(0,1,2,1)(0,1,2,1)(\bmod 3) \\
& \left(a_{1}^{2}, a_{2}^{2}, a_{3}^{2}\right)=(2,2,0)+(0,2,2) 2+(0,0,2)=(2,6,6)(2,0,0)(\bmod 3) \\
& \left(a_{1}^{3}, a_{2}^{3}\right)=(2,1)+(1,2) 2+(0,1)=(4,6)(1,0)(\bmod 3) \\
& \left(a_{1}^{4}\right)=(0)+(0) 2+(2)=(2)(2)(\bmod 3)
\end{aligned}
$$




\section{Vertex Relationships}

We've looked at how combinations of the four basic patterns can be used to represent the $3^{4}=81$ possible patterns for $n=4$, but we need to be sure that our conditions for prediction always hold. To do so, we need to take a closer look at the relationship between the leftmost and rightmost elements in the first row and the sole element of the bottom row $\left(a_{1}^{1}, a_{4}^{1}, a_{1}^{4}\right)$.

Here, basic pattern 1 becomes (gray, white, black), basic patterns 2 and 3 are (white, white, white), and basic pattern 4 is (white, gray, black). The respective modifications are (black, white, gray), (white, white, white), and (white, black, gray). Basic patterns 2 and 3 are all white, so we can exclude them from consideration. Then all we need to do is check the four possible combinations of basic patterns 1 and 2 and their modifications $((1)(2)$ and (4)(5) in Fig. 9).

$$
\begin{aligned}
& (\mathrm{G}, \mathrm{W}, \mathrm{B})+(\mathrm{W}, \mathrm{G}, \mathrm{B})=(\mathrm{G}, \mathrm{G}, \mathrm{G}), \\
& (1,0,2)+(0,1,2)=(1,1,4)(1,1,1)(\bmod 3) \\
& (\mathrm{G}, \mathrm{W}, \mathrm{B})+(\mathrm{W}, \mathrm{B}, \mathrm{G})=(\mathrm{G}, \mathrm{B}, \mathrm{W}), \\
& (1,0,2)+(0,2,1)=(1,2,3)(1,2,0)(\bmod 3) \\
& (\mathrm{B}, \mathrm{W}, \mathrm{G})+(\mathrm{W}, \mathrm{G}, \mathrm{B})=(\mathrm{B}, \mathrm{G}, \mathrm{W}), \\
& (2,0,1)+(0,1,2)=(2,1,3)(2,1,0)(\bmod 3) \\
& (\mathrm{B}, \mathrm{W}, \mathrm{G})+(\mathrm{W}, \mathrm{B}, \mathrm{G})=(\mathrm{B}, \mathrm{B}, \mathrm{B}) \\
& (2,0,1)+(0,2,1)=(2,2,2)(2,2,2)(\bmod 3)
\end{aligned}
$$

A superposition of basic patterns 1 and 4 thus maintains the relationship between the three corners and does not interfere with predictions.

We've limited discussion here to the case where $n=4$, but you can do something similar to show that the relationship between the three corners is retained in the general case $(n=10,28, \cdots)$. Just let the three corners for basic pattern 1 be (gray, white, black), those for basic patterns 2 through $n-1$ be (white, white, white), and those for basic pattern $n$ be (white, gray, black). Then it becomes necessary to consider only superpositions of basic patterns 1 and $n$, and the relation between the three vertices will be maintained, allowing the prediction.

\section{Fractal Structure}

These basic patterns are important for solving this three-color triangle problem, as the key lies in the state of the vertices in those patterns. I began to wonder 


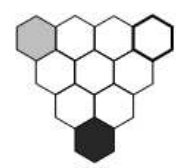

(1)

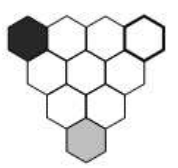

(2)

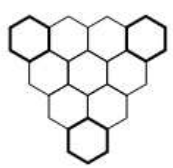

(3)

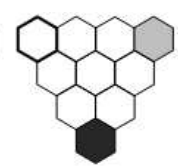

(4)

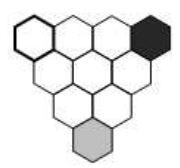

(5)

Figure 9: The three vertices of the basic patterns

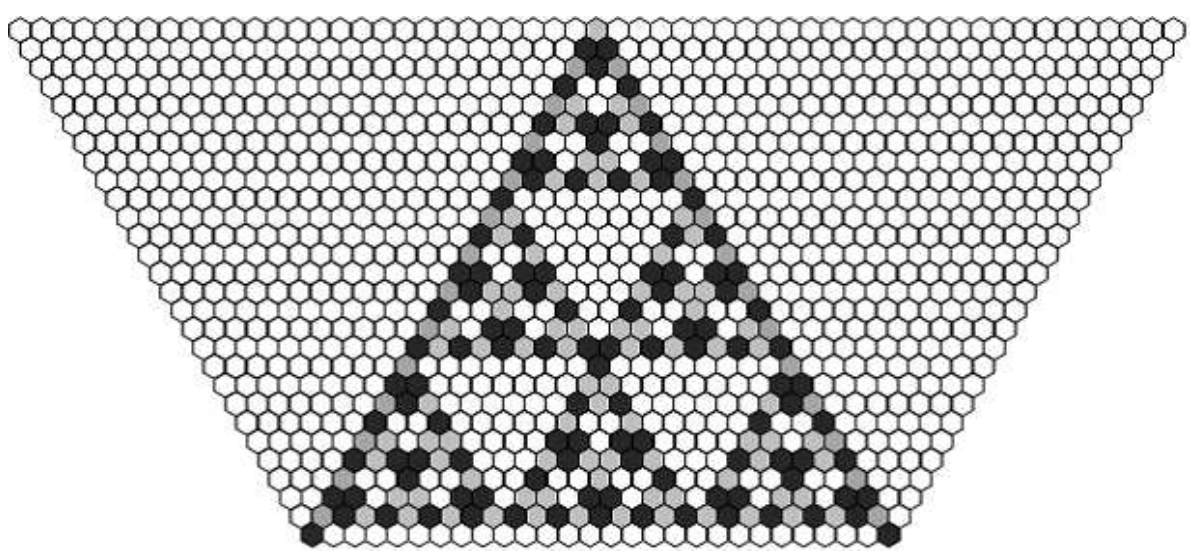

Figure 10: The all-purpose chart (for $n=2$ through $n=28$ )

whether there might be a way to efficiently draw the basic patterns for a general $n$. After some trial and error, I came up with a good idea, the all-purpose chart shown in Fig. 10. This chart lets you know at a glance the basic patterns for $n=2$ through $n=28$.

Creating the chart is simple; just start with an array of $27+1+27=55$ elements, and color each of them white except for the middle one, which is gray. After that, all that's left is to color rows 2 through 28 according to the coloring rules. Doing so leaves you with the inverted trapezoid shape in Fig. 10, which contains a self-similar equilateral triangle pattern.

This all-purpose chart contains the basic patterns for the cases of $n=2$ through $n=28$. Let's take the case of $n=4$ as an example to see how to read it. Figure 11 shows a shape extracted from the upper center part of the shape in Fig. 10. The top row in this isosceles trapezoid has 7 elements, the bottom row has 4 , and the height of the shape is 4 . The basic patterns for $n=4$ are found by extracting inverted equilateral triangles from this image, starting from the far right and moving left, one element at a time (Fig. 12). 


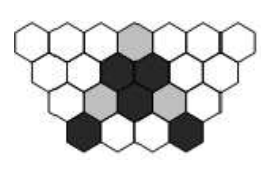

Figure 11: Basic-pattern construction chart (for $n=4$ )

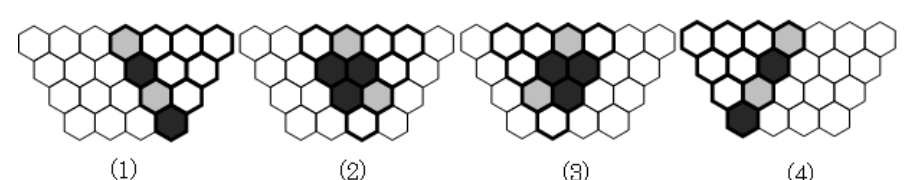

(1)

(2)

(3)

(4)

Figure 12: Basic patterns (for $n=4$ )

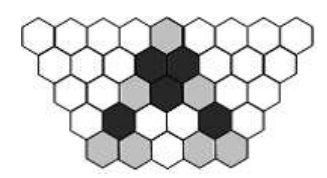

Figure 13: Basic-pattern construction chart (for $n=5)$

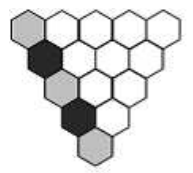

(1)

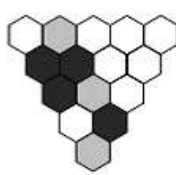

(2)

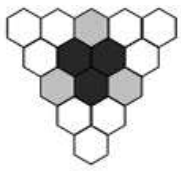

(3)

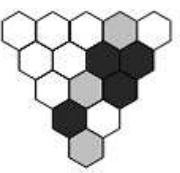

(4)

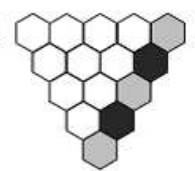

(5)

Figure 14: Basic patterns (for $n=5$ )

The basic patterns for $n=5$ are found in a similar way; extract a basicpattern construction chart like that shown in Fig. 13, and read the basic patterns off from right to left to get the results shown in Fig. 14.

Looking at the relationship between the three vertices (left, right, bottom) in the five basic patterns for $n=5$, we see that only the pattern in Fig. 14(3) fulfills the rules. We therefore already know that $n=5$ is not a solution.

It takes a little practice to become comfortable at reading basic patterns from the all-purpose chart, but once you get used to it, you can easily find the basic patterns for $n=2$ through $n=28$. The diagram also lets you search for solutions to the problem, by examining whether the three vertices of each 


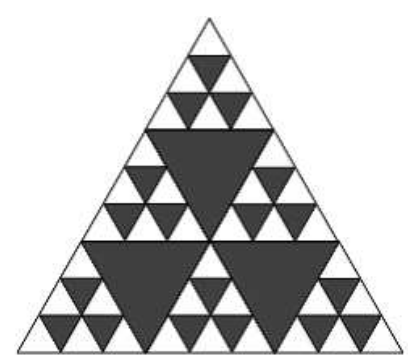

Figure 15: The Sierpiński gasket

basic pattern satisfies the rules. I invite you to do so for the solutions discussed: $n=2,4,10$, and 28 . Creating a larger all-purpose chart would allow searching for more, but once you've noticed the regularity (self-similarity) in the diagram you can predict that the next solutions will be at $n=81,244,730, \cdots$. In other words, solutions are of the form

$$
n=3^{s}+1,(s=0,1,2, \cdots)
$$

You've probably seen another image that looks very much like the allpurpose chart: the famous fractal known as the Sierpinski gasket (Fig. 15). That fractal is composed of infinitely many self-similar triangles, and is named after the Polish mathematician Waclaw Sierpinski. The all-purpose chart is a fractal shape, and its solutions have a fractal structure. This three-color triangle problem has therefore taken us on quite a trip, from Abelian groups and the superposition principle all the way to fractal geometry.

Returning to the original problem, Fig. 1 shows the case where $n=10$, and Fig. 16 shows the basic patterns for that case. Figure 17 shows the four basic patterns $(2,4,6$, and 10) that generate the triangle in Fig. 1. 


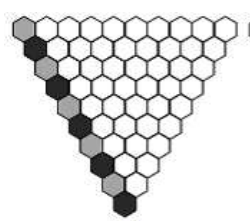

(1)

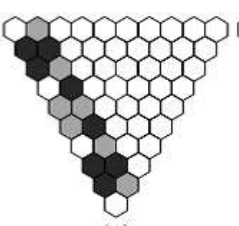

(2)

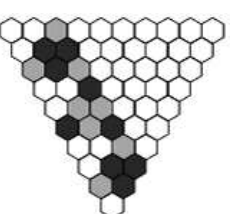

(3)

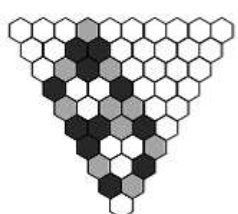

(4)

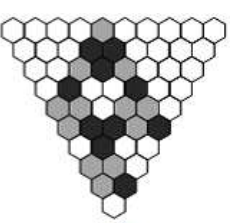

(5)

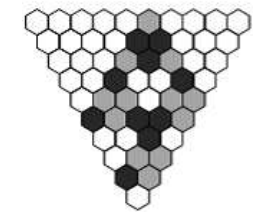

(6)

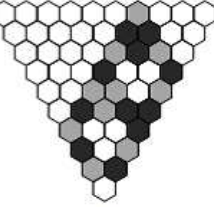

(7)

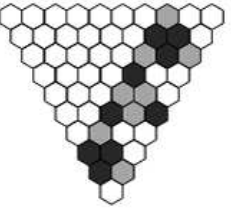

(8)

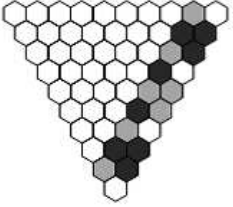

(9)

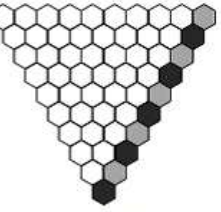

(10)

Figure 16: Basic patterns (for $n=10$ )

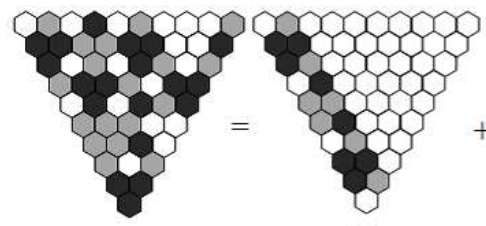

(2)

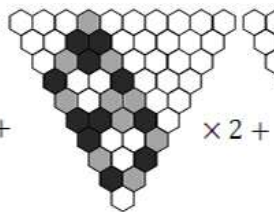

(4)

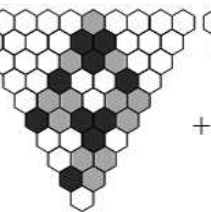

(6)

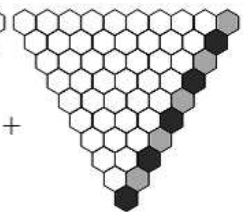

(10)

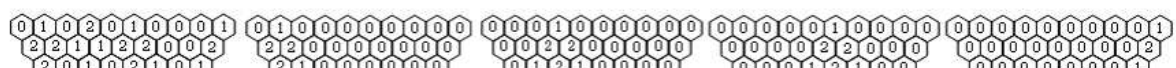

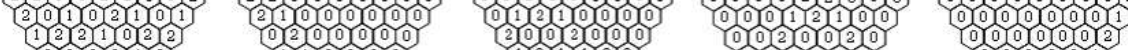

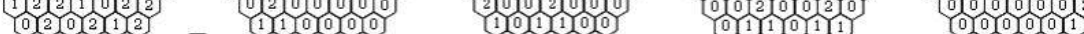

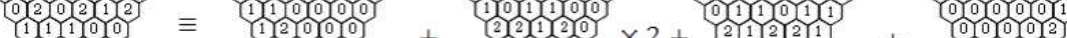
11100 110 110 (2)

Figure 17: Basic patterns for the triangle in Fig. 1 
